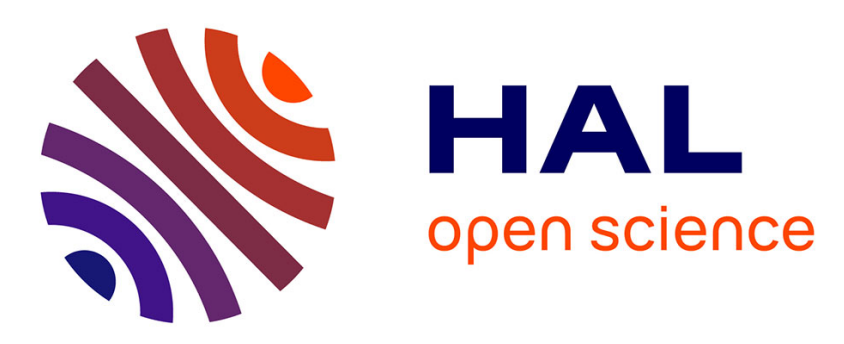

\title{
Influence des zones d'accès sur la résistance à l'état passant des transistors moyennes tensions VDMOS de puissance
}

Jean-Louis Sanchez, Henri Tranduc, T. Phan Pham, Mohamed Gharbi, P. Rossel, G. Charitat, B. Vertongen

\section{To cite this version:}

Jean-Louis Sanchez, Henri Tranduc, T. Phan Pham, Mohamed Gharbi, P. Rossel, et al.. Influence des zones d'accès sur la résistance à l'état passant des transistors moyennes tensions VDMOS de puissance. Revue de Physique Appliquée, 1985, 20 (11), pp.759-770. 10.1051/rphysap:019850020011075900 . jpa-00245391

\section{HAL Id: jpa-00245391 https://hal.science/jpa-00245391}

Submitted on 1 Jan 1985

HAL is a multi-disciplinary open access archive for the deposit and dissemination of scientific research documents, whether they are published or not. The documents may come from teaching and research institutions in France or abroad, or from public or private research centers.
L'archive ouverte pluridisciplinaire HAL, est destinée au dépôt et à la diffusion de documents scientifiques de niveau recherche, publiés ou non, émanant des établissements d'enseignement et de recherche français ou étrangers, des laboratoires publics ou privés. 
Classification

Physics Abstracts

$65.00-72.80$

\title{
Influence des zones d'accès sur la résistance à l'état passant des transistors moyennes tensions VDMOS de puissance*
}

\author{
J. L. Sanchez, H. Tranduc, T. Phan Pham, M. Gharbi, P. Rossel, G. Charitat et B. Vertongen $\left(^{+}\right)$ \\ Laboratoire d'Automatique et d'Analyse des Systèmes du C.N.R.S., \\ 7, avenue du Colonel Roche, 31077 Toulouse Cedex, France
}

$\left(^{+}\right)$La Radiotechnique, Centre Industriel de Caen, BP 6025, 14001 Caen, France

(Reçu le 18 novembre 1983, révisé le 15 mars 1985, accepté le 29 juillet 1985)

\begin{abstract}
Résumé. - Une analyse du comportement de la résistance à l'état passant $R_{\mathrm{ON}}$ du transistor DMOS de puissance est proposée. Elle tient compte en particulier de la contribution des zones d'accès au canal de conduction, à savoir le volume du drain peu dopé et la zone d'accumulation sous la grille. Pour ce faire, la dépendance expérimentale de la mobilité des électrons dans la couche accumulée en fonction du champ électrique transversal, est étudiée : le comportement est du type hyperbolique. Les limites théoriques des variations du produit « résistance ONsurface " en fonction de la tension de claquage en sont déduites. Il apparaît que la contribution de la couche accumulée est prépondérante dans le domaine des structures moyennes tensions - 100 à $600 \mathrm{~V}$ - où les performances sont directement dépendantes de la densité d'intégration et des paramètres géométriques.
\end{abstract}

\begin{abstract}
The contribution of the access regions - i.e. the drain drift bulk and the accumulated drain layer under the gate - to the ON resistance of power DMOST is studied in this paper. In particular, we analyse the effect of the reduction of the electron drift mobility in an accumulation layer as a function of the gate voltage. As a result, the theoretical limitations of the $\mathrm{ON}$ - state conductance per unit area as a function of the breakdown voltage and the geometrical parameters, are determined. It appears that the weight of the accumulated layer is of the first importance in the medium voltage range $(100 \mathrm{~V}$ to $600 \mathrm{~V})$.
\end{abstract}

\section{Introduction.}

Le développement technologique des transistors MOS pour les applications dites de puissance a permis la conception de nouveaux types de structures qui sont à la fois capables de supporter des tensions élevées à l'état bloqué et de présenter une faible chute de tension à leurs bornes lorsqu'elles transitent des courants importants à l'état passant. Ces nouvelles structures sont basées sur la technologie du drain épitaxié; les familles technologiques les plus développées à ce jour sont : i) les transistors élaborés sur les flancs de sillons gravés par attaque anisotrope du silicium (VMOS, UMOS); ii) les transistors à source et drain non coplanaires VDMOS, réalisés par le processus de double diffusion, TMOS, HEXFET, SIPMOS, TRIMOS... Afin de répondre aux exigences de fonctionnement précédemment énoncées, le facteur de qualité le plus important d'une utilisation en commutation

(*) Travail soutenu par la D.I.E.L.I. est : la caractéristique tension de claquage $V_{\mathrm{DBR}^{-}}$ résistance à l'état passant $R_{\mathrm{ON}}$.

Nous considérerons dans cet article que la résistance $\boldsymbol{R}_{\mathrm{ON}}$ d'un transistor DMOS, schématisée en coupe sur la figure 1, peut être décomposée en trois éléments :

i) La résistance $R_{\mathrm{ch}}$ du canal d'inversion,

ii) La résistance $R_{\mathrm{a}}$ correspondant à la région d'accès au canal, de la zone du drain épitaxié située sous la grille,

iii) La résistance $R_{\mathrm{d}}$ dite de $d r i f t$ correspondant à la zone de drain épitaxié dans laquelle s'écoulent les porteurs jusqu'au contact même du drain.

Dans une publication antérieure, nous avions proposé un calcul de la résistance du canal et d'une partie de la résistance du volume $N^{-}$[1]. Nous avions négligé, dans cette étude, l'effet de la présence de la couche accumulée. Les paramètres d'étude les plus significatifs pris en compte avaient été ceux de la géométrie de la structure. Il avait été montré tout d'abord que le facteur le plus important, pour améliorer les perfor- 


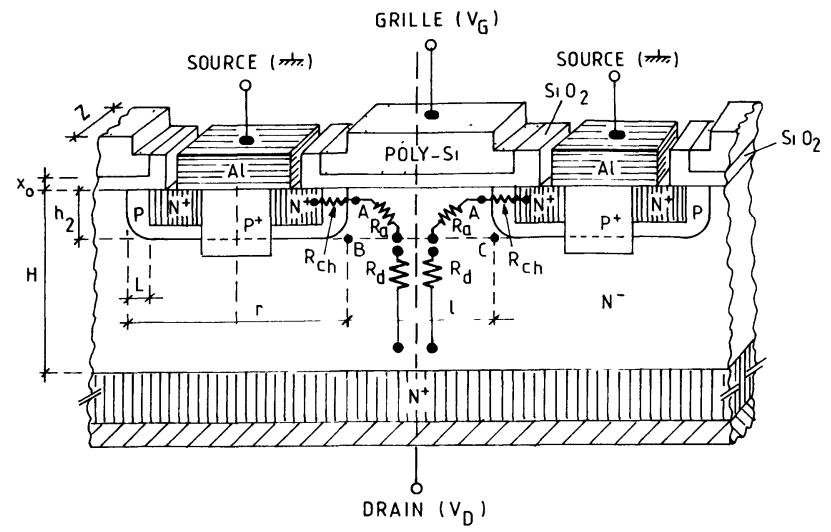

Fig. 1. - Coupe schématique d'une cellule de transistor DMOS de puissance, localisation des résistances $R_{\mathrm{ch}}, R_{\mathrm{a}}$ et $R_{\mathrm{d}}$.

[Cross-section of the $\mathrm{N}$-channel DMOS transistor elementary cells. Location of the channel resistance $R_{\mathrm{ch}}$, the access resistance $R_{\mathrm{a}}$ and the drift resistance $R_{\mathrm{d}}$.]

mances des structures basses tensions - claquage inférieur à $100 \mathrm{~V}$ - dont la résistance passante est liée essentiellement à la valeur de $R_{\mathrm{ch}}$, est celui de l'accroissement de la densité d'intégration. Pour ce faire, il est nécessaire de choisir judicieusement la configuration géométrique des cellules élémentaires constituant la source du transistor. De plus, les limites asymptotiques - $\left(R_{\mathrm{ON}} \cdot S\right)^{-1}$ en fonction de la tension de claquage $V_{\mathrm{DBR}}-$ des performances des transistors fonctionnant, soit en basses tensions $(<100 \mathrm{~V})$, soit en hautes tensions ( $>700 \mathrm{~V}$ ), ont pu être établies.

L'objet du présent article est de compléter cette analyse en proposant une méthode de calcul de la résistance d'accès $R_{\mathrm{a}}$ qui inclut l'influence de la couche accumulée dont le poids devient important dans le domaine des moyennes tensions $(100$ à $600 \mathrm{~V})$. Pour y parvenir, il est nécessaire de prendre en compte le mécanisme de conduction dans la zone accumulée. Aussi une étude particulière visant à déterminer l'effet du champ transverse sur la mobilité dans cette zone accumulée sera proposée. Nous préciserons ainsi la limite complète du compromis "résistance à l'état passant-tension de claquage ", en utilisant le modèle de la résistance d'accès $R_{\mathrm{a}}$ et les limites asymptotiques basses et hautes tensions publiées antérieurement [1].

\section{Le modèle de la résistance de la zone d'accès.}

La résistance $R_{\mathrm{ON}}$ est définie comme étant le rapport entre tension drain et courant drain lorsque cette tension drain-source $V_{\mathrm{D}}$ tend vers zéro. Comme nous l'avons déjà indiqué, elle se décompose en trois éléments :

i) la résistance $R_{\mathrm{ch}}$ du canal d'inversion qui se situe dans la diffusion $P$ sous la grille, ii) la résistance d'accès $R_{\mathrm{a}}$ correspondant à la région d'accès, iii) la résistance $R_{\mathrm{d}}$ dite de drift correspondant à la zone de drain épitaxié dans laquelle les porteurs défocalisent jusqu'au contact de drain.

$$
R_{\mathrm{ON}}=R_{\mathrm{ch}}+R_{\mathrm{a}}+R_{\mathrm{d}} .
$$

La localisation de ces zones a pu être précisée par une étude bidimensionnelle - résolution des équations de Laplace, de Poisson et de continuité - par différences finies - dont un exemple de résultat est présenté sur la figure 2 .

Dans le cadre de cette étude, nous nous limiterons à l'analyse de la contribution de $R_{\mathrm{a}}$. Les expressions de $R_{\mathrm{ch}}$ et $R_{\mathrm{d}}$ déjà étudiées par ailleurs [1] seront rappelées dans le paragraphe 4.

La résistance d'accès $R_{\mathrm{a}}$ est définie comme étant la résistance qui relie le point $A$ - fin du canal d'inversion sous la grille - au plan BC (Fig. 1), considéré comme étant l'équipotentielle qui délimite la zone de défocalisation des lignes de courant dans le corps du semiconducteur $N^{-}$. Sa valeur est contrôlée par deux mécanismes dont les effets répartis sont liés à :

i) la présence de la couche accumulée, induite par la tension grille-source lorsqu'elle est supérieure à la tension drain-source. Cette couche apparaît à la surface de la région semiconductrice $N^{-}$qui est recouverte par la grille.

ii) la résistance du volume du matériau $N^{-}$situé au dessous de cette couche accumulée, jusqu'à une

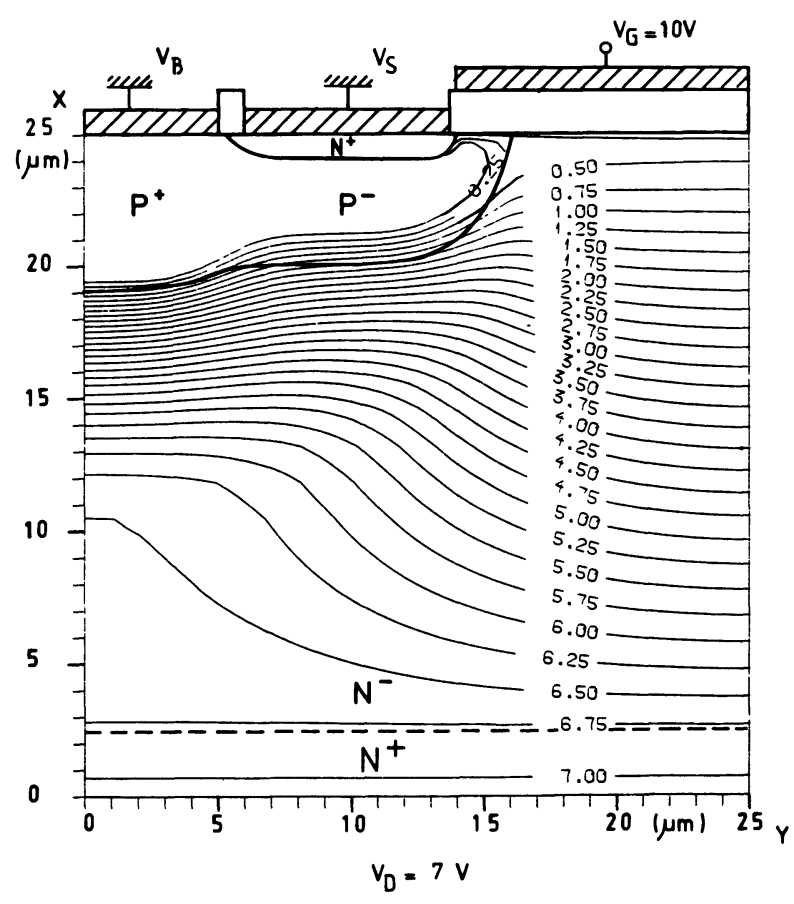

Fig. 2. - Chutes de tension dans le semiconducteur d'un transistor MOS haute tension (1/2 cellule). Etude bidimensionnelle des équipotentielles en régime de conduction $\left(V_{\mathrm{G}}=10 \mathrm{~V}, V_{\mathrm{D}}=7 \mathrm{~V}\right)$.

[Two-dimensional calculation : equipotential plots for half a VDMOS elementary cell with the $\mathrm{ON}$-state conditions : $V_{\mathrm{G}}=10 \mathrm{~V}, V_{\mathrm{D}}=7 \mathrm{~V}$.] 
profondeur $h_{2}$ équivalente à la profondeur de jonction du caisson de type $P$.

1.1 LE SCHÉMA À ÉLÉMENTS RÉPARTIS DE LA ZONE D'ACCÈS. - En vue de décrire les propriétés de cette résistance d'accès $R_{\mathrm{a}}$ - lorsque la grille est polarisée positivement par rapport à la source dans le cas du transistor à canal $N$ - nous représentons la zone $N^{-}$ du semiconducteur correspondant à $R_{\mathrm{a}}$, par le schéma distribué dans les deux directions $x$ et $y$ (Fig. 3). Selon la direction $y$, on prend en compte l'effet de modification de la résistance superficielle de la couche accumulée par la tension grille-drain. Entre l'interface $\mathrm{Si}_{-} \mathrm{SiO}_{2}$ et la limite matérialisée par la ligne BC(Fig. 1), on prend en compte l'effet de la résistance volumique du semiconducteur. Dans le cas de faibles tensions drain-source $V_{\mathrm{D}}$, on considère que le schéma équivalent est celui de la figure 3 qui traduit une représentation bidimensionnelle par une approche deux fois unidimensionnelle, verticale et horizontale.

La figure 4 représente les résistances différentielles $\mathrm{d} R$ et $\mathrm{d} r$ associées dans une tranche du semiconducteur de largeur dy. En explicitant le courant à partir de la charge totale $q N$ des porteurs dans la zone accumulée, la résistance différentielle $\mathrm{d} R$ s'exprime par :

$$
\frac{\mathrm{d} R}{\mathrm{~d} y}=\frac{1}{\mu_{\mathrm{acc}} \cdot Z \cdot q \cdot N}
$$

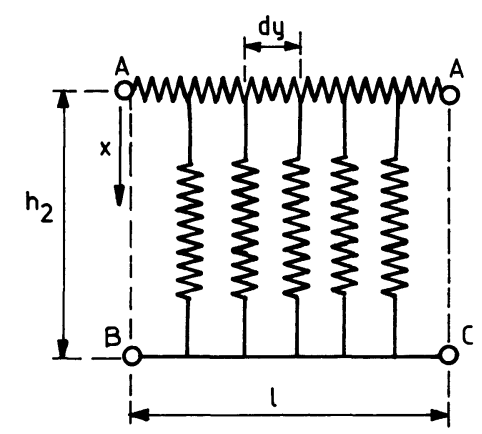

Fig. 3. - Schéma équivalent de la résistance d'accès $R_{\mathrm{a}}$. [Equivalent circuit of the access resistance $R_{\mathrm{a}}$ ]

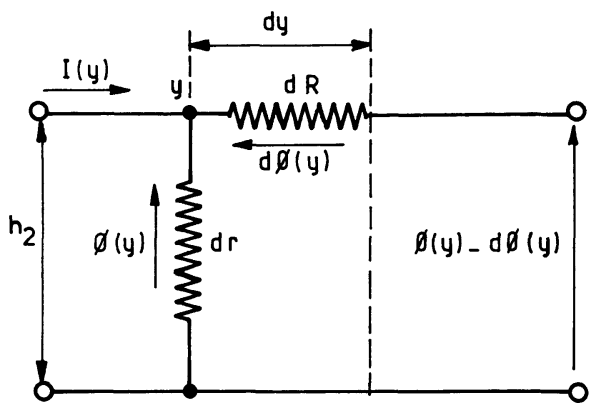

Fig. 4. - Schéma équivalent d'une tranche différentielle de résistance d'accès $R_{\mathrm{a}}$.

[Equivalent circuit of an elementary cell of $R_{\mathrm{a}}$.] avec

$$
q N=-C_{\mathrm{ox}}\left[V_{\mathrm{G}}^{\prime}-\frac{k T}{q} \cdot \operatorname{Ln}\left(1+\frac{q}{k T} \cdot \frac{V_{\mathrm{G}}^{\prime 2}}{\phi_{\mathrm{B}}}\right)\right] .
$$

L'expression de cette charge est obtenue en intégrant l'équation de neutralité dans la tranche différentielle en régime d'accumulation [2]. Rappelons que dans ces relations $\mu_{\text {acc }}$ représente la mobilité des électrons dans la couche accumulée, $Z$ la largeur totale du canal, $V_{G}^{\prime}$ la tension effective appliquée sur la grille, $C_{\text {ox }}$ la capacité par unité de surface de la couche d'oxyde de grille, $k T / q$ le potentiel thermodynamique, $\phi_{\mathrm{B}}$ le potentiel électrostatique du volume $N^{-}$égal à $2 . q N_{\mathrm{D}} \cdot \varepsilon_{\mathrm{Si}} \cdot \varepsilon_{0} \cdot C_{\mathrm{ox}}^{-2}: \varepsilon_{\mathrm{Si}} \cdot \varepsilon_{0}$ représentant la permittivité du silicium et $N_{\mathrm{D}}$ le dopage de la couche $N^{-}$.

La résistance $\mathrm{d} r$ d'une tranche différentielle $\mathrm{d} y \mathrm{de}$ volume s'exprime sous la forme :

$$
\mathrm{d} r=\frac{\rho \cdot h_{2}}{Z \cdot \mathrm{d} y}
$$

où $\rho$ représente la résistivité du semiconducteur $N^{-}$ supposée indépendante des coordonnées $(x, y)$ et $h_{2}$ la profondeur de jonction du caisson $P$.

1.2 EXPRESSION DE LA RÉSISTANCE D'ACCÈS $R_{\mathrm{a}}$. - En vue de déterminer l'expression de la résistance d'accès $\boldsymbol{R}_{\mathrm{a}}$ lorsque la grille est polarisée positivement par rapport à la source, nous traitons la région d'accès du transistor comme une ligne passive identique à celle représentée sur les figures 3 et 4 .

En désignant par $I(y)$ le courant à l'abscisse $y$ et par $\mathrm{d} I(y)$ le courant élémentaire circulant dans une résistance $\mathrm{d} r$ de volume élémentaire, le comportement d'une telle ligne se traduit par les relations suivantes :

$$
\begin{aligned}
\mathrm{d} \phi(y) & \simeq I(y) \cdot \mathrm{d} R \\
\mathrm{~d} I(y) & =\frac{\phi(y)}{\mathrm{d} r} .
\end{aligned}
$$

En tenant compte de la symétrie de la structure représentée sur la figure 1 et en prenant l'origine des $y$ au milieu du segment BC (Fig. 3), la théorie des lignes fournit les expressions du courant et du potentiel :

$$
\begin{aligned}
& \phi(y)=2 \pi \cdot \cosh (\sqrt{\alpha \beta} \cdot y) \\
& I(y)=2 \pi \sqrt{\frac{\alpha}{\beta}} \cdot \sinh (\sqrt{\alpha \beta} \cdot y)
\end{aligned}
$$

où $\pi$ est une constante qui dépend de la valeur du potentiel aux limites :

$$
\phi(-l / 2)=\phi(l / 2)
$$

$l$ étant la distance interdiffusion $P$.

La résistance d'accès est définie par :

$$
R_{\mathrm{a}}=\frac{\phi(l / 2)}{I(l / 2)}
$$


Par suite, en explicitant les valeurs de $I(y)$ et de $\phi(y)$, pour une structure de périmètre de canal $Z$, la résistance $R_{\mathrm{a}}$ s'exprime par :

$$
R_{\mathrm{a}}=\frac{2 \cdot h_{2}}{q \cdot \mu_{\mathrm{n}} \cdot N_{\mathrm{D}} \cdot Z \cdot l} \cdot \lambda
$$

où le coefficient $\lambda$ est défini par :

$$
\begin{aligned}
\lambda=\frac{l}{2} \sqrt{\frac{q \mu_{\mathrm{n}} N_{\mathrm{D}}}{\mu_{\mathrm{acc}} \cdot C_{\mathrm{ox}} \cdot h_{2} \cdot f\left(V_{\mathrm{G}}\right)}} \times \\
\times \frac{1}{\tanh \left[\frac{l}{2} \sqrt{\frac{q \mu_{\mathrm{n}} N_{\mathrm{D}}}{\mu_{\mathrm{acc}} \cdot C_{\mathrm{ox}} \cdot h_{2} \cdot f\left(V_{\mathrm{G}}\right)}}\right]}
\end{aligned}
$$

avec

$$
f\left(V_{\mathrm{G}}\right)=V_{\mathrm{G}}^{\prime}-\frac{k T}{q} \cdot \operatorname{Ln}\left(1+\frac{q}{k T} \cdot \frac{V_{\mathrm{G}}^{\prime 2}}{\phi_{\mathrm{B}}}\right)
$$

$\mu_{\mathrm{n}}$ représente la mobilité des porteurs dans le volume du semiconducteur de dopage $N_{\mathrm{D}}$.

L'expression (9) montre que la résistance $R_{\mathrm{a}}$ peut être considérée comme le produit de la résistance d'un barreau semiconducteur - de section $Z . l / 2$, de longueur $h_{2}$ et de dopage $N_{\mathrm{D}}$ - par le coefficient $\lambda$ qui dépend du dopage, des dimensions géométriques, de la tension grille et de la valeur de mobilité $\mu_{\text {acc }}$.

Les paramètres de mobilité des électrons dans une couche accumulée sont présentés dans le paragraphe suivant.

2. Mobilité des porteurs dans une couche accumulée. Réduction de mobilité due au champ transversal.

Afin de connaître les paramètres traduisant l'effet de réduction de mobilité due au champ transversal, dans la région accumulée d'un transistor DMOS, nous avons réalisé des structures MOS qui permettent d'étudier le fonctionnement en régime accumulé. De telles structures ainsi que le principe de mesure de mobilité sont décrits en annexe. Nous présentons sur la figure 5 des exemples de variations de mobilité dans cette couche en fonction de la tension effective appliquée sur la grille $V_{G}^{\prime}$.

La courbe 6 représentant l'évolution de l'inverse de $\mu_{\text {acc }}$ en fonction de $V_{G}^{\prime}$, permet de vérifier que la variation de mobilité des électrons, dans la couche accumulée, en fonction du champ transverse, obéit à une loi expérimentale de forme hyperbolique pour les valeurs de tension de grille supérieures à 2 volts - identique à celle déjà proposée pour l'inversion [3]; nous l'écrivons par suite sous la forme phénoménologique suivante :

$$
\mu_{\mathrm{acc}} \mid \begin{aligned}
& =\frac{\mu_{0 \mathrm{a}}}{1+\frac{V_{\mathrm{G}}^{\prime}}{\Lambda}} \\
& V_{\mathrm{G}}^{\prime}>2 V .
\end{aligned}
$$

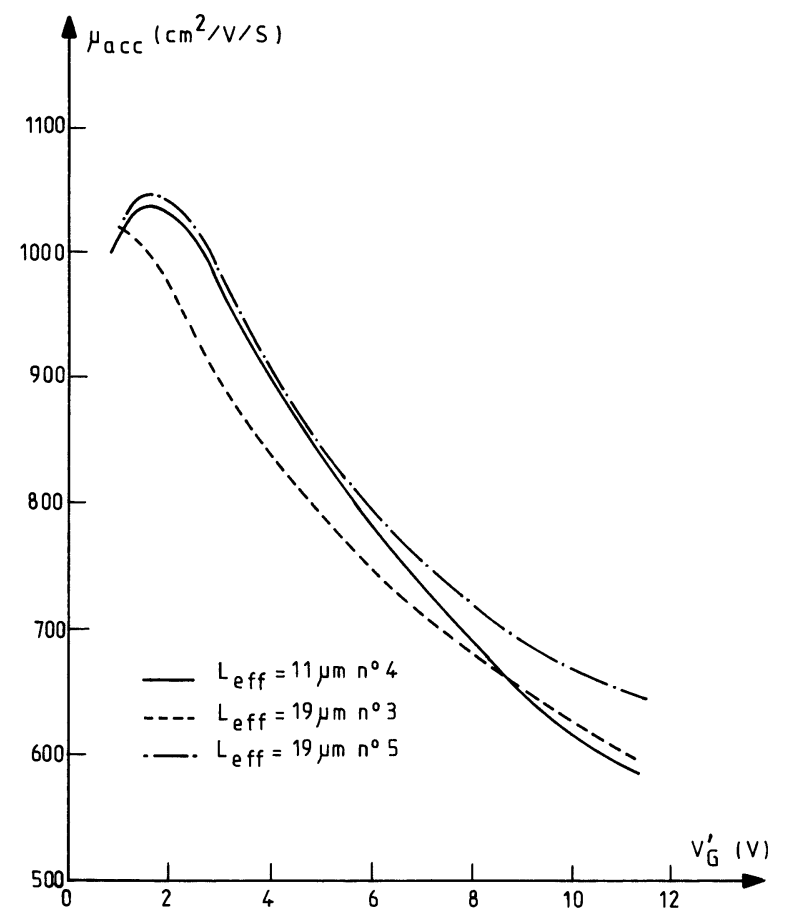

Fig. 5. - Variation de la mobilité dans la couche accumulée en fonction de la tension effective appliquée sur la grille.

[Experimental variations of the drift mobility in the accumulated layer as a function of the effective gate voltage.]

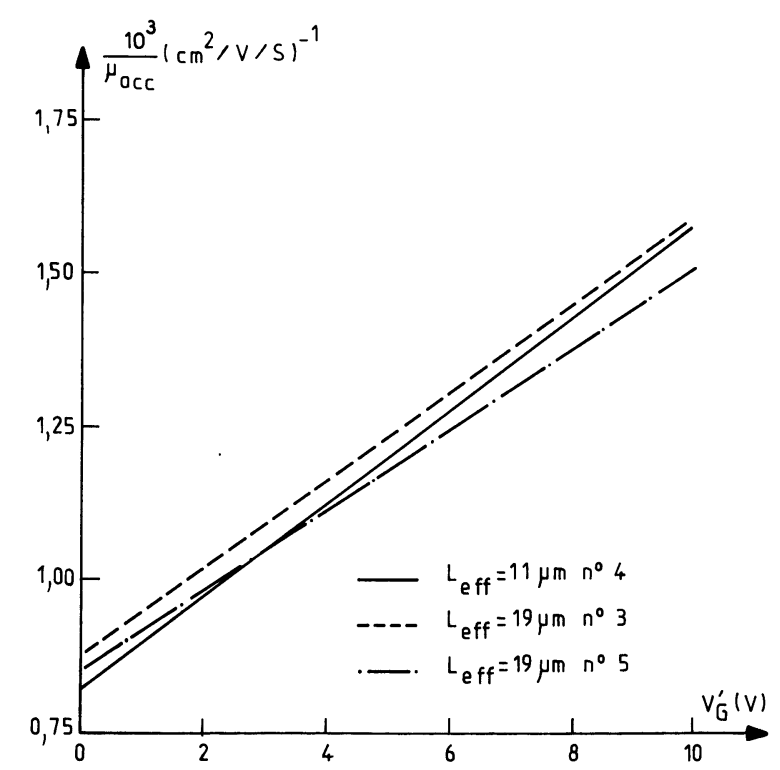

Fig. 6. - Mise en évidence du mode d'évolution hyperbolique de la mobilité dans la couche accumulée.

No 4. $-L_{\text {eff }}=11 \mu \mathrm{m} ; \Lambda=11 \quad \mathrm{~V} ; \mu_{0 \mathrm{a}}=1200 \mathrm{~cm}^{2} / \mathrm{V} / \mathrm{s}$ No 3. $-L_{\text {eff }}=19 \mu \mathrm{m} ; \Lambda=12,2 \mathrm{~V} ; \mu_{0 \mathrm{a}}=1140 \mathrm{~cm}^{2} / \mathrm{V} / \mathrm{s}$ No $5 .-L_{\text {eff }}=19 \mu \mathrm{m} ; \Lambda=12,8 \mathrm{~V} ; \mu_{0 \mathrm{a}}=1170 \mathrm{~cm}^{2} / \mathrm{V} / \mathrm{s}$

[Comparison of the experimental drift mobility behaviour in an accumulated layer with a hyperbolic law.] 
Dans cette relation, $\mu_{0 \mathrm{a}}$ représente la mobilité à champ faible des électrons dans la couche accumulée, $\Lambda$ un coefficient de réduction de mobilité due au champ transversal et $V_{\mathrm{G}}^{\prime}$ la tension effective appliquée sur la grille. Les valeurs de $\mu_{0 \mathrm{a}}$, sont données par extrapolation hyperbolique à $V_{G}^{\prime}=0$, les pentes des droites représentées sur la figure 6 permettent de déduire les valeurs des coefficients $\Lambda$ correspondants

$$
\begin{aligned}
\mu_{0 \mathrm{a}} & \simeq 1170 \mathrm{~cm}^{2} / \mathrm{V} / \mathrm{s} \\
\Lambda & \simeq 12 \mathrm{~V} .
\end{aligned}
$$

Des études récentes [4] ont fourni des résultats équivalents du point de vue des ordres de grandeur.

\section{Evolution de la résistance d'accès en fonction de la tenue en tension. Influence de l'épaisseur d'oxyde.}

Les relations liant les paramètres relatifs à la couche épitaxiée, épaisseur, dopage, mobilité dans le volume en fonction de la tension de claquage drain-source $V_{\text {DBR }}$, ont été établies par le passé au Laboratoire [1]. Il a été montré que le couple épaisseur-dopage, permettant de minimiser la résistance volumique du barreau $N^{-}$dans la direction verticale, s'exprime par :

$$
\begin{aligned}
\left(H-h_{2}\right) \text { opt } & =1,8 \times 10^{-8} V_{\mathrm{DBR}}^{1,2}(\mathrm{MKSA}) \\
N_{\mathrm{D}} \mathrm{opt} & =1,94 \times 10^{24} V_{\mathrm{DBR}}^{-1,4}(\mathrm{MKSA}) .
\end{aligned}
$$

Rappelons également que l'expression de la mobilité $\mu_{\mathrm{n}}$ dans le volume peut être représentée par la relation numérique proposée par $\mathrm{C} . \mathrm{Hu}$ [5] qui relie $\mu_{\mathrm{n}}$ à la tension de claquage.

$$
\mu_{\mathrm{n}}=0,07 . V_{\mathrm{DBR}}^{0,1}(\mathrm{MKSA}) \text {. }
$$

3.1 EVOLUTION DU COEFFICIENT $\lambda$ EN FONCTION DE LA TENUE EN TENSION. EXPRESSION ASYMPTOTIQUE. En introduisant l'expression analytique de la mobilité des électrons dans la couche accumulée, déterminée dans le paragraphe précédent, on obtient pour le coefficent $\lambda(10)$ :

$\lambda=\frac{l}{2} \sqrt{\frac{q \cdot N_{\mathrm{D}} \cdot a \cdot\left(\Lambda+V_{\mathrm{G}}^{\prime}\right)}{C_{\mathrm{ox}} \cdot h_{2} \cdot \Lambda \cdot V_{\mathrm{G}}^{\prime}}} \times$

$$
\times \frac{1}{\tanh \left[\frac{l}{2} \sqrt{\frac{q \cdot N_{\mathrm{D}} \cdot a \cdot\left(\Lambda+V_{\mathrm{G}}^{\prime}\right)}{C_{\mathrm{ox}} \cdot h_{2} \cdot \Lambda \cdot V_{\mathrm{G}}^{\prime}}}\right]}
$$

où $a$ représente le rapport $\mu_{\mathrm{n}} / \mu_{0 \mathrm{a}}$. La mobilité $\mu_{0 \mathrm{a}}$ à champ faible étant du même ordre de grandeur que la mobilité dans le volume, le coefficient $a$ sera pris égal à l'unité. En ce qui concerne le coefficient de réduction de mobilité $\Lambda$ dans la couche accumulée, nous prendrons $\Lambda=12 \mathrm{~V}$ pour une épaisseur d'oxyde de grille de $1000 \AA$.

Compte tenu des relations (13) et (14), la dépendance du coefficient $\lambda$ en fonction de la tenue en tension est

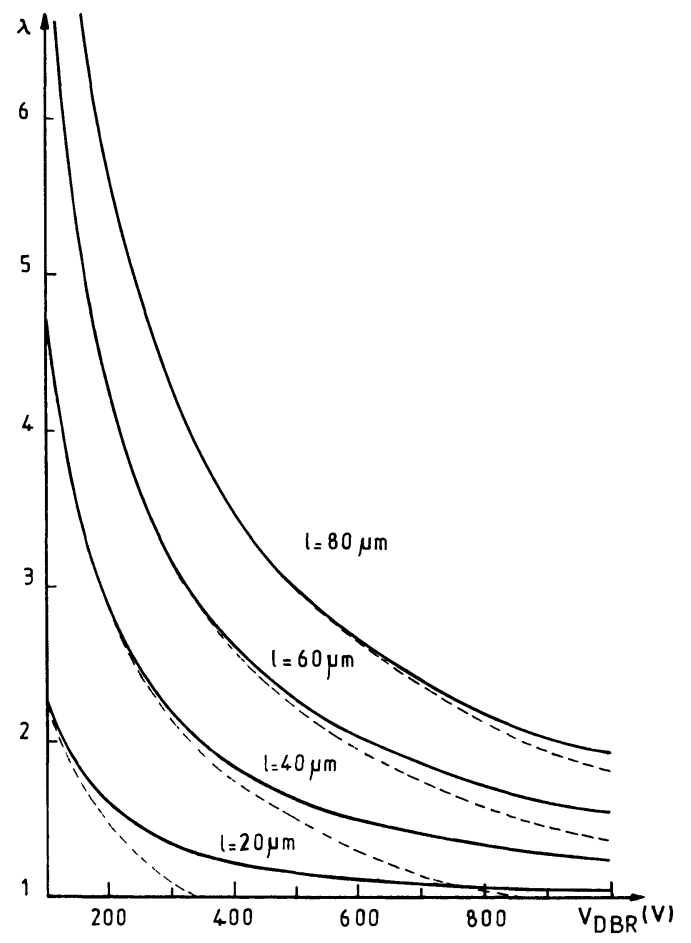

Fig. 7. - Variation de $\lambda$ en fonction de la tension de claquage — solution exacte (relation (15)) --- solution asymptotique (relation (16)) $h_{2}=5 \mu \mathrm{m}, V_{\mathrm{G}}^{\prime}=10 \mathrm{~V}, a=1, \Lambda=$ $12 V$, épaisseur d'oxyde $1000 \AA$.

[Variations of the access resistance coefficient $\lambda$ as a function of the breakdown voltage. - expression (15) --- asymptotic, expression (16).]

illustrée par la figure 7. Nous distinguons deux domaines de variations :

i) lorsque la tension $V_{\text {DBR }}$ est faible, $\lambda$ tend vers l'expression asymptotique suivante :

$$
\lambda_{\text {asym }}=\frac{l}{2} \cdot \sqrt{\frac{q \cdot N_{\mathrm{D}} \cdot\left(\Lambda+V_{\mathrm{G}}^{\prime}\right)}{C_{\mathrm{ox}} \cdot h_{2} \cdot \Lambda \cdot V_{\mathrm{G}}^{\prime}}}
$$

ii) pour des tensions de claquage élevées, le coefficient $\lambda$ tend vers 1 .

\subsection{EVolution DE LA CONDUCTANCE DE LA ZONE} D'ACCÈS EN FONCTION DE LA TENUE EN TENSION. - En nous plaçant dans l'hypothèse d'une structure optimale pour laquelle $Z . l=S$ (Annexe 2), la conductance par unité de surface a pour expression :

$$
\begin{aligned}
\left(R_{\mathrm{a}} S\right)^{-1}=\frac{q \mu_{\mathrm{n}} N_{\mathrm{D}}}{l \cdot h_{2}} & \cdot \sqrt{\frac{C_{\mathrm{ox}} \cdot h_{2} \cdot \Lambda \cdot V_{\mathrm{G}}^{\prime}}{q N_{\mathrm{D}}\left(\Lambda+V_{\mathrm{G}}^{\prime}\right)}} \times \\
& \times \tanh \left[\frac{l}{2} \sqrt{\frac{q N_{\mathrm{D}}\left(\Lambda+V_{\mathrm{G}}^{\prime}\right)}{C_{\mathrm{ox}} \cdot h_{2} \cdot \Lambda \cdot V_{\mathrm{G}}^{\prime}}}\right]
\end{aligned}
$$

où $h_{2}$ représente la profondeur de jonction du caisson $P$. 
Dans la plupart des composants VDMOS réalisés actuellement, cette valeur est comprise entre 3 et $5 \mu \mathrm{m}$. La figure 8 représente les courbes $\left(R_{\mathrm{a}} . S\right)^{-1}$ pour différentes valeurs de $l$ avec une valeur de $h_{2}$ fixée à $5 \mu \mathrm{m}$, correspondant au cas le plus classique. Les valeurs des autres paramètres sont les suivantes : $a=1, x_{0}=1000 \AA, V_{\mathrm{G}}^{\prime}=10 \mathrm{~V}$ et $\Lambda=12 \mathrm{~V}$.

Dans le domaine des moyennes tensions $-V_{\mathrm{DBR}}<$ $600 \mathrm{~V}$ - nous pouvons utiliser la valeur asymptotique de $\lambda$ relation (16); l'expression asymptotique de $\left(R_{\mathrm{a}} S\right)^{-1}$ devient alors :

$$
\left(R_{\mathrm{a}} S\right)^{-1}=\frac{q \mu_{\mathrm{n}} N_{\mathrm{D}}}{l \cdot h_{2}^{1 / 2}} \sqrt{\frac{C_{\mathrm{ox}} \cdot \Lambda \cdot V_{\mathrm{G}}^{\prime}}{q N_{\mathrm{D}}\left(\Lambda+V_{\mathrm{G}}^{\prime}\right)}} .
$$

Compte tenu des valeurs des paramètres $V_{\mathrm{G}}^{\prime}=10 \mathrm{~V}$, $\Lambda=12 \mathrm{~V}, x_{0}=1000 \AA$, ainsi que des relations (13) et (14), on obtient :

$$
\left(R_{\mathrm{a}} S\right)^{-1}=\frac{1,69}{l \cdot h_{2}^{1 / 2}} \cdot V_{\mathrm{DBR}}^{-0,6}(\mathrm{MKSA}) .
$$

Cette limite asymptotique reportée sur la figure 8 est confondue avec la courbe correspondant à la relation complète (17), dans tout le domaine des moyennes tensions. En tant qu'élément partiel de conclusion, il apparaît que la relation (18) qui traduit la limite de la quantité $\left(R_{\mathrm{a}} S\right)^{-1}$, est originale et plus rigoureuse que celles proposées antérieurement. Elle est particulièrement bien adaptée à l'étude de l'influence de l'épaisseur de l'oxyde recouvrant la région intercellulaire sur la résistance d'accès.

3.3 INFLUENCE DE L'ÉPAISSEUR D'OXYDE INTERCELLULAIRE. - Dans le but d'obtenir des temps de commutation faibles, de l'ordre de 5 à $20 \mathrm{~ns}$, les travaux actuels s'orientent vers l'étude de structures VDMOS présentant un oxyde plus épais sur la région d'accès [6, 7] (Fig. 9). Cette configuration permet de minimiser la capacité de réaction grille-drain. Dans ce paragraphe, nous nous proposons d'analyser l'influence de l'augmentation de l'épaisseur d'oxyde sur la valeur de la conductance de la région d'accès. En prenant $Z . l / S=1$ (Annexe 2) et compte tenu des relations (13) et (14), l'expression asymptotique (18) prend la forme suivante :

$$
\left(R_{\mathrm{a}} S\right)^{-1}=\frac{39}{l \cdot h_{2}^{1 / 2}} \cdot V_{\mathrm{DBR}}^{-0,6} \cdot \sqrt{\frac{C_{\mathrm{ox}} \cdot \Lambda \cdot V_{\mathrm{G}}^{\prime}}{\left(\Lambda+V_{\mathrm{G}}^{\prime}\right)}}(\mathrm{MKSA}) .
$$

En prenant comme hypothèse que le coefficient de réduction de mobilité $\Lambda$ dans la couche accumulée varie proportionnellement avec l'épaisseur d'oxyde comme c'est le cas pour le régime d'inversion, la valeur de $\Lambda$ qui est de $12 \mathrm{~V}$ pour une épaisseur d'oxyde de $1000 \AA$, vaudra $60 \mathrm{~V}$ pour une épaisseur de $5000 \AA$, et $120 \mathrm{~V}$ pour $10000 \AA$.

La figure 10 représente, par exemple, les variations de la conductance par unité de surface $\left(R_{\mathrm{a}} S\right)^{-1}$ en

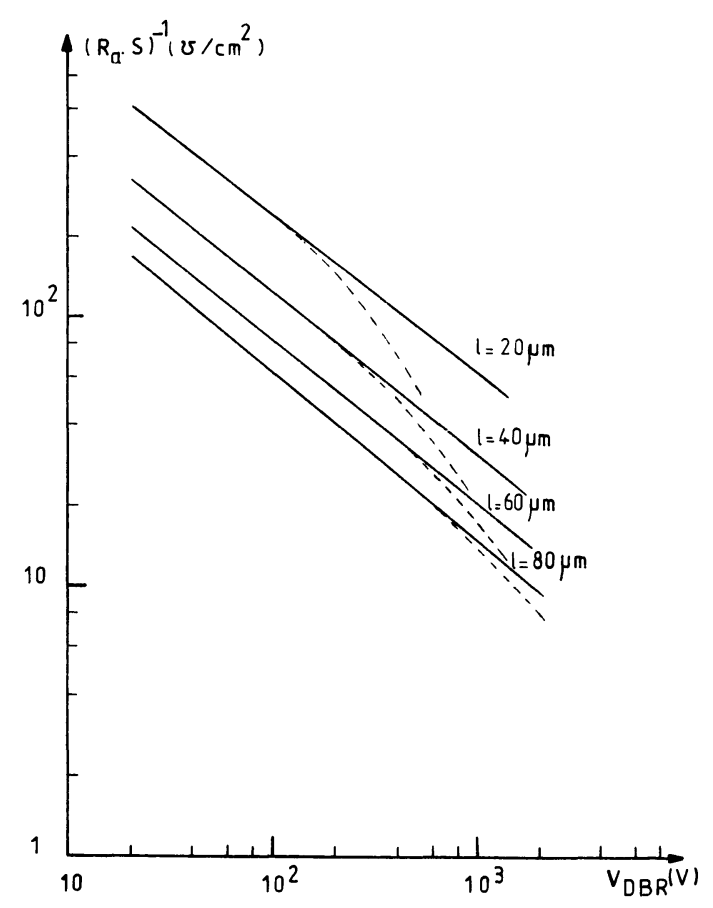

Fig. 8. - Evolution de $\left(R_{\mathrm{a}} S\right)^{-1}-V_{\mathrm{DBR}}-$ courbe asymptotique (relation (18)) --- (relation (17)) $h_{2}=5 \mu \mathrm{m}$, épaisseur d'oxyde $1000 \AA, A=12 \mathrm{~V}, V_{\mathrm{G}}^{\prime}=10 \mathrm{~V}$.

[Variations of the access conductance $\left(R_{\mathrm{a}} S\right)^{-1}$ as a function of the breakdown voltage, --- expression (17)—_ asymptotic expression (18).]

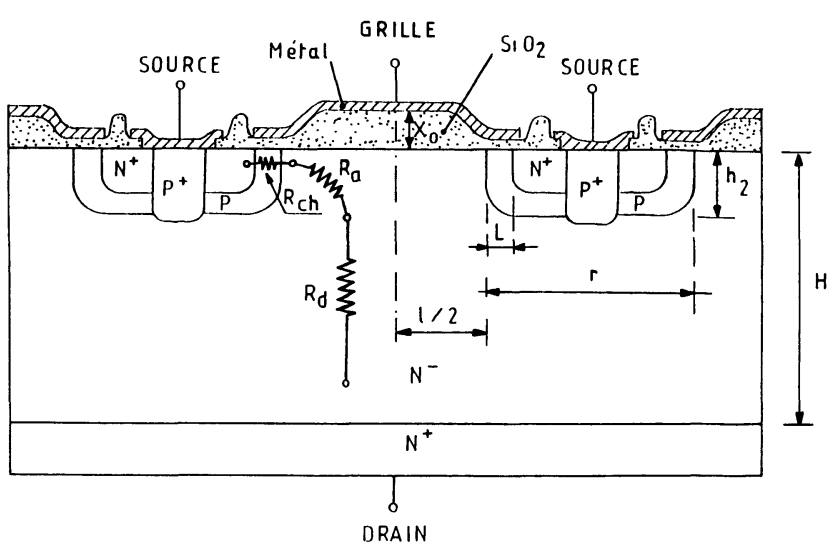

Fig. 9. - Coupe schématique d'une structure VDMOS avec deux niveaux d'oxyde de grille.

[Cross-section of a VDMOS transistor with two oxide thickness levels.]

fonction de l'épaisseur d'oxyde $x_{0}$ recouvrant la région d'accès, lorsque les autres paramètres sont fixés à des valeurs typiques moyennes : $l=40 \mu \mathrm{m}$, $h_{2}=5 \mu \mathrm{m}$ et $V_{G}^{\prime}=10 \mathrm{~V}$. Il apparaît que la conductance de la région d'accès se dégrade notablement lorsque l'épaisseur de la couche d'oxyde augmente. 


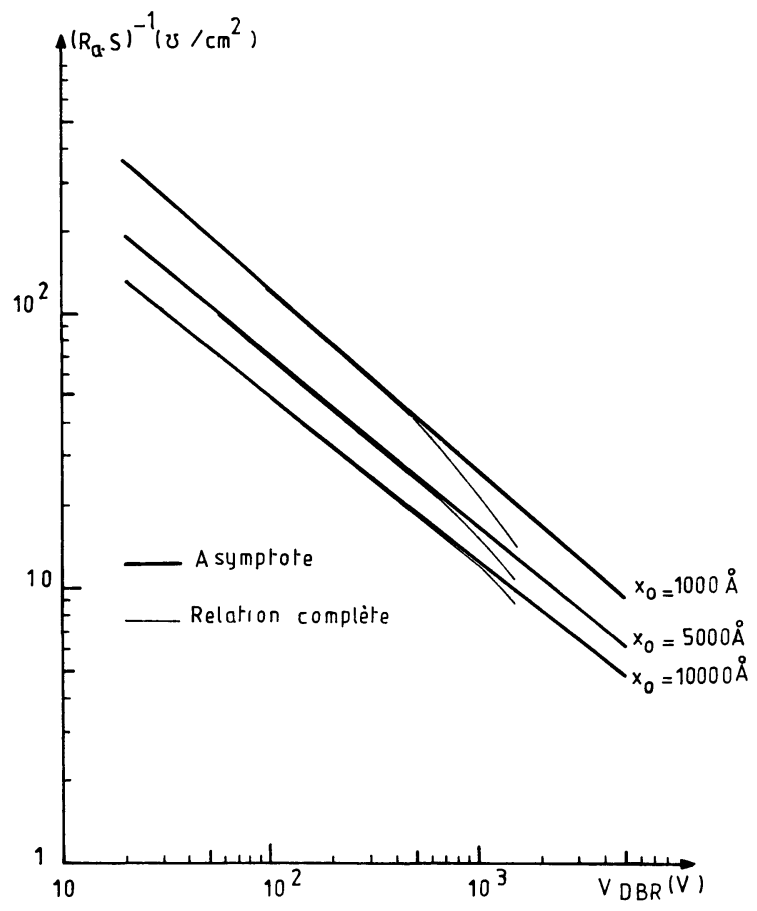

Fig. 10. - Influence de l'épaisseur d'oxyde intercellulaire sur la conductance par unité de surface de la zone d'accès.

$$
V_{\mathrm{G}}^{\prime}=10 \mathrm{~V}, l=40 \mu \mathrm{m}, \quad h_{2}=5 \mu \mathrm{m} \text {. }
$$

[Variations of the access conductance as a function of the breakdown voltage with the thickness of the oxide over the accumulation drain region as parameter.]

Cette conductance chute, pour une tension de claquage donnée, d'un facteur voisin de deux entre un oxyde de $1000 \AA$ et un oxyde de $10000 \AA$. Toutefois, et en contre-partie, la diminution corrélative de la capacité grille-drain permet d'obtenir un "gain » en temps de commutation qui pourra s'avérer intéressant pour certaines applications spécifiques. Des exemples de résultats obtenus en commutation sur de telles structures sont proposés par ailleurs [6,7].

\section{Compromis $\left(R_{\mathrm{ON}} \cdot S\right)^{-1}-V_{\mathrm{DBR}}$}

Afin de déterminer, à présent, les limites asymptotiques de la quantité globale $\left(R_{\mathrm{ON}} \cdot S\right)^{-1}$ en fonction de la tension $V_{\mathrm{DBR}}$ dans toute la gamme de tension, nous reportons les limites des trois conductances $\left(R_{\mathrm{ch}} \cdot S\right)^{-1}$, $\left(R_{\mathrm{d}} \cdot S\right)^{-1}$ et $\left(R_{\mathrm{a}} S\right)^{-1}$ qui la composent.

4.1 LA RÉSISTANCE DU CANAL DE CONDUCTION. L'expression de la résistance du canal $R_{\mathrm{ch}}$ est déduite de l'équation du courant d'inversion du transistor MOS. Cette résistance est égale au rapport entre la tension aux bornes du canal de conduction et le courant de drain à faible tension drain. La conductance, par unité de surface correspondante, apparaît comme étant indépendante de la tension de claquage; elle vaut [1] :

$$
\begin{aligned}
\left(R_{\mathrm{ch}} \cdot S\right)^{-1}=\mu_{0} & \cdot \frac{\varepsilon_{0} \cdot \varepsilon_{\mathrm{ox}}}{x_{0}} \times \\
& \times \psi \cdot \frac{V_{\mathrm{G}}^{\prime}}{V_{\mathrm{G}}^{\prime}+\psi}(L \cdot l)^{-1}\left(\frac{Z . l}{S}\right)
\end{aligned}
$$

où $\varepsilon_{0} \cdot \varepsilon_{\mathrm{ox}}$ représente la permittivité de l'oxyde, $x_{0}$ son épaisseur, $L$ la longueur du canal de conduction, $Z$ sa largeur, $\mu_{0}$ la mobilité à champ faible des électrons dans la couche d'inversion, $\psi$ le potentiel de réduction de mobilité due au champ transversal, $V_{G}^{\prime}$ la tension effective de grille.

Les valeurs numériques des paramètres qui $y$ interviennent, sont :

i) $1000 \AA$ pour l'épaisseur d'oxyde, ce qui correspond à ce jour à la valeur normalisée réalisée sur les structures de puissance;

ii) la tension de grille effective $V_{G}^{\prime}$ est normalisée à $10 \mathrm{~V}$;

iii) la mobilité $\mu_{0}$ et le potentiel de réduction de mobilité $\psi$ sont pris égaux à $650 \mathrm{~cm}^{2} / \mathrm{V} / \mathrm{s}$ et $25 \mathrm{~V}$ respectivement.

4.2 LA RÉSISTANCE DE VOLUME DU DRAIN PEU DOPÉ. En ce qui concerne la résistance de drift $R_{\mathrm{d}}$, nous utiliserons une expression analytique déjà proposée par B. De Moraes [8] dont le calcul est basé sur l'utilisation de la méthode de transformation conforme [9].

$$
R_{\mathrm{d}}=\frac{2 \rho}{\pi \cdot Z} \cdot \operatorname{Argsinh}\left\{\frac{\sinh \left(\frac{\pi\left(H-h_{2}\right)}{l(1+r / l)}\right)}{\sin \left(\frac{\pi}{2(1+r / l)}\right)}\right\}
$$

qui peut s'écrire sous une forme que l'on identifie à :

$$
R_{\mathrm{d}}=\frac{2 \rho\left(H-h_{2}\right)}{Z(l+r)} \cdot \Gamma .
$$

Le terme $\frac{2 \rho\left(H-h_{2}\right)}{Z(l+r)}$ correspond à la résistance $R_{\mathrm{vol}}$ du barreau semiconducteur de section $Z \frac{(l+r)}{2}$, de longueur $\left(H-h_{2}\right)$ et de résistivité $\rho$.

Le coefficient $\Gamma$ qui traduit le phénomène de défocalisation des lignes de courant est donné par la relation suivante :

$\Gamma=\frac{l}{H-h_{2}} \cdot \frac{(1+r / l)}{\pi} \cdot \operatorname{Argsinh}\left\{\frac{\sinh \left(\frac{\pi\left(H-h_{2}\right)}{l(1+r / l)}\right)}{\sin \left(\frac{\pi}{2(1+r / l)}\right)}\right\}$.

Dans le cas d'une structure optimisée $(Z . l / S=1$ et $r=l$ ) compte tenu des expressions $(12,13,14)$, 
liant respectivement l'épaisseur, le dopage et la mobilité de volume de la couche épitaxiée à la tension de claquage $V_{\mathrm{DBR}}$, nous reportons sur la figure 11 les limites de $\left(R_{\mathrm{d}} \cdot S\right)^{-1}$ pour différentes valeurs de $l$ comprises entre 20 et $100 \mu \mathrm{m}$. Nous remarquons d'une part, que ces courbes sont situées entre deux limites extrêmes, à savoir :

$$
\text { i) }\left(R_{\mathrm{d}} \cdot S\right)^{-1}=12 \times 10^{11} V_{\mathrm{DBR}}^{-2,5}(\mathrm{MKSA}) \text {. }
$$

Cette limite correspond à la résistance totale du volume - notée $R_{\mathrm{vol}}$ - atteinte pour une défocalisation totale des lignes de courant; dans ce cas, $\Gamma$ est égal à l'unité.

$$
\begin{array}{r}
\left(R_{\mathrm{d}} \cdot S\right)^{-1}=6 \times 10^{11} V_{\mathrm{DBR}}^{-2,5}(\mathrm{MKSA})= \\
=\frac{\left(R_{\mathrm{vol}} \cdot S\right)^{-1}}{2}
\end{array}
$$

Dans l'hypothèse, $r=l$, cette limite correspond au double de la résistance de volume $-R_{\text {vol }}-$ pour laquelle il n'est pas tenu compte de la défocalisation; dans ce cas, $\Gamma$ est égal à deux.

D'autre part, il apparaît qu'à l'intérieur de ces limites, les courbes $\left(R_{\mathrm{d}} S\right)^{-1}$ tracées pour des valeurs de $l$ comprises entre 20 et $100 \mu \mathrm{m}$, sont quasiment confondues. Par suite, la modification des dimensions géométriques apporte assez peu d'amélioration sur la contribution de la résistance $R_{\mathrm{d}}$.

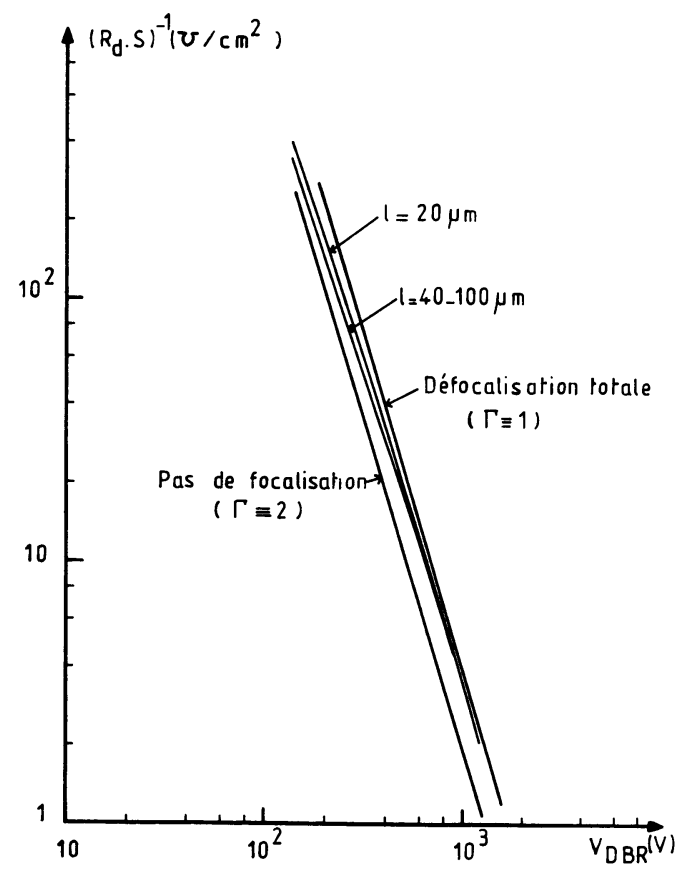

Fig. 11. - Evolution de $\left(R_{\mathrm{d}} \cdot S\right)^{-1}-V_{\mathrm{DBR}}$. Limites asymptotiques théoriques.

[Variations of the drift conductance as a function of the breakdown voltage.]
4.3 Evolution DE LA CONDUCTANCE À L'ÉTAT PASSANT EN FONCTION DE LA TENUE EN TENSION. - La composition de ces diverses caractéristiques asymptotiques conduit à définir dans le plan $\left(R_{\mathrm{ON}} \cdot S\right)^{-1}$ $V_{\mathrm{DBR}}$, les courbes limites théoriques en dessous desquelles se situent les valeurs de la quantité $\left(R_{\mathrm{ON}} \cdot S\right)^{-1}$ de toutes les structures de transistors MOS (Fig. 12).

Les principaux paramètres sont figés aux meilleures valeurs de $\mu_{0}$ et $\psi$ que nous ayons mesuré sur orientation $\langle 100\rangle$ par les techniques et méthodes explicitées dans la publication [10] (Tableau I).

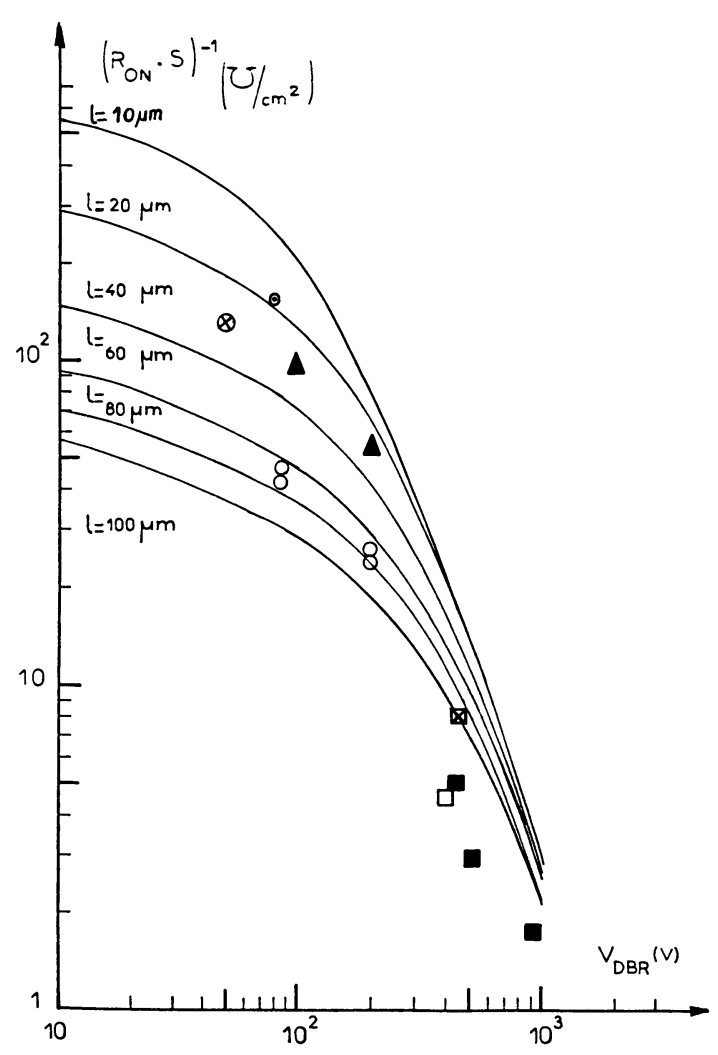

Fig. 12. - Etat de l'art $\left(R_{\mathrm{ON}} \cdot S\right)^{-1}-V_{\mathrm{DRR}}-V D M O S$ avec oxyde intercellulaire mince $(1000 \AA), L=2 \mu \mathrm{m}$, $\psi=25 \mathrm{~V}, \mu_{0}=650 \mathrm{~cm}^{2} / \mathrm{V} / \mathrm{s}, \quad V_{\mathrm{G}}^{\prime}=10 \mathrm{~V}, \Lambda=12 \mathrm{~V}$. Théorie : - - - .

Expérience :

$\otimes$ SIEMENS BUZ 71

O IR HEXFET

$\triangle$ RTC TRIMOS

$\triangle$ HPWR

- TMOS MOTOROLA

LAAS Oxyde mince $\mathrm{Cr}$ $\odot$ TMOS III MOTOROLA

En ce qui concerne les structures expérimentales, $l$ varie de $12,5 \mu \mathrm{m}$ (dispositifs basses tensions) à $50 \mu \mathrm{m}$ (dispositifs hautes tensions).

[Variations of the output conductance by unit area as a function of the breakdown voltage, with the inter-cell distance as parameter. Theoretical and experimental results.] 
Tableau I. - Valeurs expérimentales des paramètres électriques utilisés dans l'expression de la résistance du canal.

\begin{tabular}{|c|c|c|c|c|c|c|}
\hline Paramètre & $\mu_{0}\left(\mathrm{~cm}^{2} / \mathrm{V} / \mathrm{s}\right)$ & $x_{0}(\AA)$ & $\psi(\mathrm{V})$ & $\Lambda(\mathrm{V})$ & $V_{\mathrm{G}}^{\prime}(\mathrm{V})$ & $L(\mu \mathrm{m})$ \\
\hline Valeur utilisée & 650 & 1000 & 25 & 12 & 10 & 2 \\
\hline
\end{tabular}

Rappelons que, bien entendu, l'étude correspond à des structures idéales optimales [1] en ce qui concerne : i) la forme des cellules - triangles ou carrés ou hexagones - ii) les valeurs du dopage et de l'épaisseur de la couche épitaxiale, iii) les profondeurs de diffusion $N$ et $P$. Rappelons en particulier que les hypothèses i) d'épaisseur de diffusion $\boldsymbol{P}$ ou $\boldsymbol{P}^{+}$négligeable devant l'épaisseur d'épitaxie $N^{-}$, ii) d'un rapport de répétition $r / l$, égal à l'unité pour les carrés ou les hexagones ou égal à $\sqrt{3}$ pour les triangles peuvent ne pas être réalisables dans le cas de certaines structures basses tensions fortement densifiées [1].

On notera, par rapport aux résultats des études antérieures [1], d'une part, que la valeur de la résistance d'accès $R_{\mathrm{a}}$ est de première importance dans la contribution à la résistance passante en moyennes tensions et d'autre part, que la limite de la contribution de la résistance $R_{\mathrm{ch}}$ est évaluée avec une bien meilleure précision.

4.3.1 Influence de la polarisation de grille. - La figure 13 illustre l'effet d'une modification de la tension effective de grille $V_{\mathrm{G}}^{\prime}$; la limite théorique $\left(R_{\mathrm{ON}_{\infty}} \cdot S\right)^{-1}$ correspondant à une tension de grille effective tendant vers l'infini, est représentée uniquement à titre indicatif. Cette tension $V_{G}^{\prime}$ influe notablement sur la résistance de sortie dans les structures basses ou moyennes tensions, puisque, dans ces cas, elle module fortement les densités de charge dans les zones inversées ou accumulées. En hautes tensions, la valeur de la tension grille a peu d'influence car la résistance est quasiment imposée par le volume $N^{-}$ du semiconducteur.

4.3.2 Influence du pas de répétition des cellules. La figure 12 met en évidence l'influence du pas de répétition $l$ sur les performances électriques des structures classiques à épaisseur d'oxyde de grille uniforme :

i) dans le domaine des basses et aussi des moyennes tensions, la densification de la géométrie des cellules permet d'augmenter la conductance totale par unité de surface;

ii) par contre, pour des tensions supérieures à $600 \mathrm{~V}$, la résistance à l'état passant tend vers la résistance de volume et la densification des cellules semble ne pas apporter d'amélioration sur la résistance à l'état passant $R_{\mathrm{ON}}$. Toutefois, des travaux complémentaires ont permis de montrer que le courant maximum transmissible par unité de surface dépend de la densité d'intégration [11] et qu'il existe une taille de cellule optimisée.

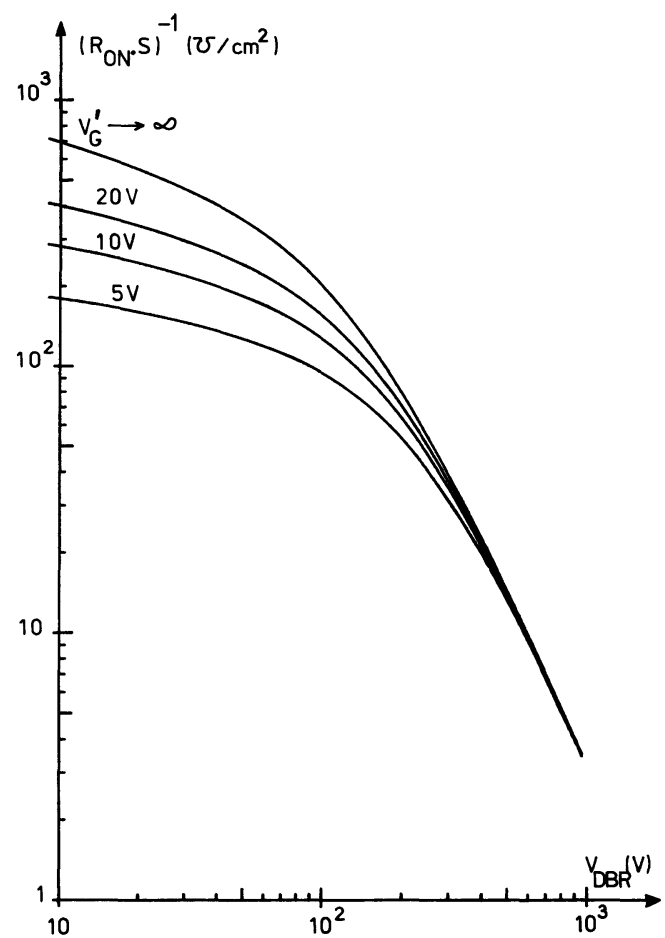

Fig. 13. - Conductance par unité de surface en fonction de la tension de claquage, pour différentes valeurs de tension effective de grille $V_{G}^{\prime}$

$$
\begin{aligned}
& R_{\mathrm{ch}}\left\{\begin{array}{l}
\mu_{0}=650 \mathrm{~cm}^{2} / \mathrm{V} / \mathrm{s} \\
\psi=25 \mathrm{~V} \\
L=2 \mu \mathrm{m} \\
l=20 \mu \mathrm{m} \\
x_{0}=1000 \AA
\end{array}\right. \\
& R_{\mathrm{a}}\left\{\begin{array}{l}
\Lambda=12 \mathrm{~V} \\
x_{0}=1000 \AA \\
a=1
\end{array}\right.
\end{aligned}
$$

[Variations of the output conductance by unit area as a function of the breakdown voltage, with the gate voltage as parameter.]

4.3.3 Cas des structures à deux niveaux d'oxyde. Le cas des structures à deux niveaux d'oxyde, l'un mince sur la couche inversée, l'autre épais sur la couche accumulée, est présenté sur la figure 14. La dégradation qui résulte de la présence de cet oxyde " multi-niveaux » sur la résistance totale bas niveau, par rapport au cas de l'oxyde uniforme, est de l'ordre de $30 \%$ pour un composant optimisé à $400 \mathrm{~V}$, lorsque l'épaisseur d'oxyde sur la zone d'accès passe de 1000 


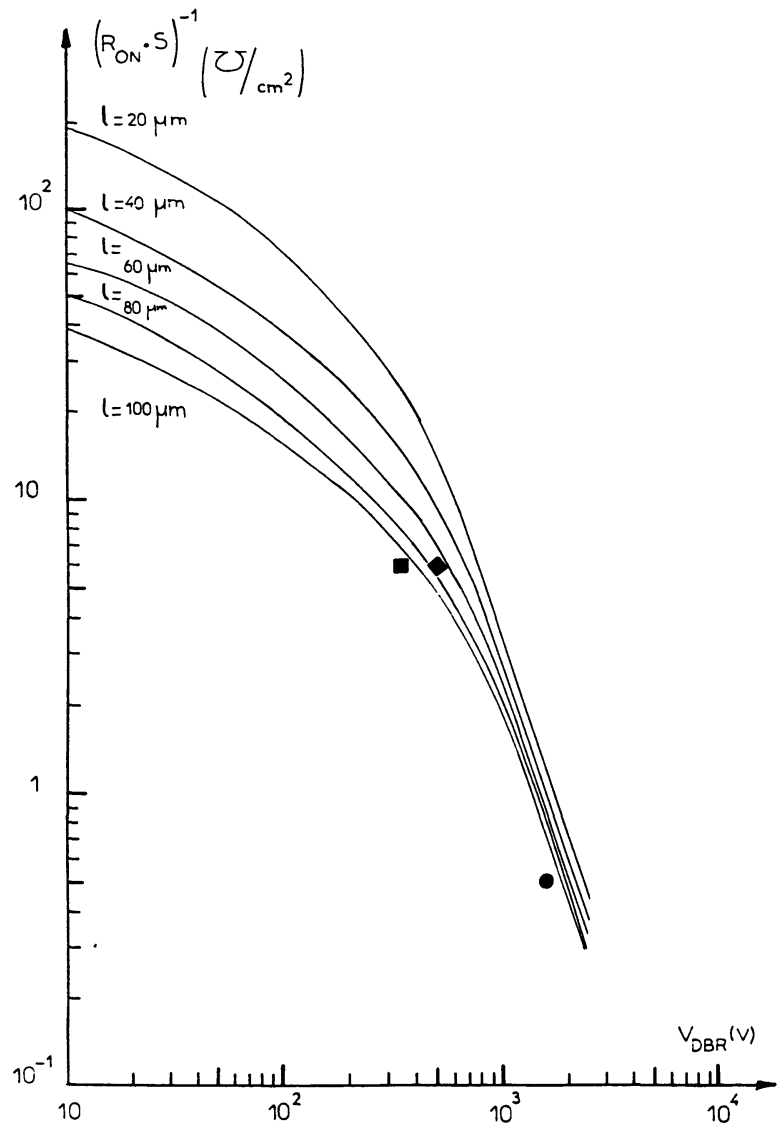

Fig. 14. - Etat de l'art $\left(R_{\mathrm{ON}} \cdot S\right)^{-1}-V_{\mathrm{DBR}}$, VDMOS avec oxyde intercellulaire épais (10000 $\AA$ ), oxyde de grille de $1000 \AA, V_{G}^{\prime}=10 \mathrm{~V}$

Théorie

$$
\begin{gathered}
\left\{\begin{array}{l}
\mu_{0}=650 \mathrm{~cm}^{2} / \mathrm{V} / \mathrm{s} \\
\psi=25 \mathrm{~V} \\
L=2 \mu \mathrm{m} \\
R_{\mathrm{ch}}=1000 \AA \\
x_{0}=120 \mathrm{~V}
\end{array}\right. \\
R_{\mathrm{a}}\left\{\begin{array}{l}
\Lambda=10000 \AA \\
x_{0}=1 \\
a=1
\end{array}\right.
\end{gathered}
$$

Expérience :

- YOSHIDA [6]

STG MOSFET [7]

- Nos résultats $(r=l=58 \mu \mathrm{m}, \rho=10 \Omega$.cm).

[Variations of the output conductance by unit area as a function of the breakdown voltage, for the VDMOST's with two oxide levels : $1000 \AA$ and $10000 \AA$. Theoretical and experimental results.]

à $10000 \AA$ A Ceci est dû essentiellement à la diminution du poids de la zone accumulée dans la valeur de la résistance de la zone d'accès.

L'intérêt de ces structures réside dans le fait qu'elles présentent une capacité d'entrée et une capacité de réaction drain-grille réduites sensiblement d'un facteur deux.

4.3.4 Etat de l'art des performances $\left(R_{\mathrm{ON}}-V_{\mathrm{DBR}}\right)$. Pour être complet, nous avons comparé les résultats théoriques aux valeurs expérimentales relevées sur la plupart des structures développées à ce jour par divers laboratoires et qui nous semblent être les plus significatives (Figs. 12, 14). Il apparaît tout d'abord, par comparaison à des résultats publiés antérieurement, que la recherche de nouveaux types de structures et l'optimisation de certaines dimensions géométriques ont permis d'améliorer au cours du temps les performances du transistor MOS de puissance. D'autre part, le gain en basses ou en moyennes tensions est lié essentiellement à l'accroissement de la densité d'intégration; dans ce cadre, il est certain que des progrès seront réalisés dans le futur grâce à la réduction des géométries.

\section{Conclusion}

Dans cet article, nous avons complété l'étude théorique du compromis $R_{\mathrm{ON}}-V_{\mathrm{DBR}}$ en introduisant l'effet de la résistance d'accès $R_{\mathrm{a}}$ qui tient compte à la fois de l'effet dû au volume du semiconducteur et de la présence de la couche accumulée sous la grille. Pour calculer avec précision les valeurs de cette contribution, nous avons pris en compte la dépendance expérimentale de la mobilité dans la couche accumulée en fonction du champ transverse. De cette étude, il apparaît que l'influence de la couche accumulée sur la résistance $R_{\mathrm{a}}$ est surtout importante dans le domaine des moyennes tensions. Dans cette gamme de tensions $\left(V_{\mathrm{DBR}}<600 \mathrm{~V}\right)$, il a été montré que la diminution des dimensions géométriques entraîne une diminution corrélative de la résistance $R_{\mathrm{ON}}$. D'autre part, la dégradation de la résistance à l'état passant due à la présence d'un oxyde épais sur la zone d'accès, dans les structures à capacité d'entrée réduite, a pu être précisée. Pour des tensions de claquage plus élevées $\left(V_{\mathrm{DBR}}>600 \mathrm{~V}\right)$, la résistance $R_{\mathrm{ON}}$ est principalement liée à la résistance de volume, et la réduction des dimensions géométriques n'a pas d'effet sur la valeur de la résistance passante. Seules, de nouvelles technologies aptes à diminuer la valeur de cette résistance de volume permettent d'abaisser la valeur de $R_{\mathrm{ON}}$. C'est ainsi que la modulation de la conductivité dans la zone épitaxiée des nouveaux dispositifs appelés COMFET [12] ou GEM ou " redresseur synchrone » [13] permet de réduire la valeur de la résistance à l'état passant d'un facteur de l'ordre de dix, et l'on se retrouve ainsi, en fait, ramené au même cas d'optimisation de la géométrie que pour les dispositifs MOS basse tension. La contrepartie est, dans ce dispositif bipolaire, une dégradation des performances dynamiques - temps de commutation ou fréquence de transition - d'un facteur du même ordre de grandeur.

\section{Annexe 1. - Mesure de la mobilité des porteurs dans la couche accumulée.}

Pour déterminer la variation de mobilité des électrons dans une couche accumulée en fonction du champ électrique transverse, on utilise des structures dites 
transistor MOS « à accumulation " dont la coupe schématique est représentée sur la figure A.1. Ces dispositifs sont réalisés sur substrat épitaxié $N^{-} P^{+}$, la source et le drain sont de type $N$, la conduction s'effectue par porteurs majoritaires. Les dispositifs réalisés ont un dopage $N_{\mathrm{D}}$ de $2 \times 10^{15}$ at. $\mathrm{cm}^{-3}$, une longueur effective entre source et drain $L_{\mathrm{ef} f}$ valant soit $7 \mu \mathrm{m}$, soit $11 \mu \mathrm{m}$, soit $19 \mu \mathrm{m}$, une épaisseur d'oxyde de $1000 \AA$ et une largeur $Z$ de $3 \mathrm{~mm}$.

A. 1 PRINCIPE DE LA déTERmination de la MOBILITÉ. - Le courant de drain $I_{\mathrm{D}}$ est la somme de trois courants : i) un courant de volume noté $I_{1}$ qui est le courant du barreau semiconducteur, de section $h . Z$ et de longueur $L$, situé entre source et drain, ii) le courant $I_{2}$ dû aux porteurs majoritaires présents dans la couche accumulée, iii) un courant de fuite noté $I_{\mathrm{F}}$ qui s'écoule entre source et drain à travers le volume du semiconducteur $N^{-}$situé sous les jonctions de source et de drain.

La figure A. 2 représente un exemple d'une caractéristique $I_{\mathrm{D}}\left(V_{\mathrm{G}}\right)$ à faible tension drain-source, obtenue avec ces composants. Sur ce type de courbe, J. S. T. Huang et G. W. Taylor [14] ont montré que la valeur de la tension de grille $V_{G}$, qui correspond au changement de pente de la caractéristique $I_{\mathrm{D}}\left(V_{\mathrm{G}}\right)$, est égale à la valeur de la tension de bande plate $V_{\text {FB }}$. Pour des tensions de grille supérieures à $V_{\mathrm{FB}}$, il y a création d'une couche accumulée sous la grille; l'expression théorique du courant d'accumulation $I_{2}$ correspondant est alors :

$$
I_{2}=\frac{Z}{L_{\text {eff }}} \cdot \mu_{\mathrm{acc}} \cdot C_{\mathrm{ox}} \cdot \int_{0}^{V_{\mathrm{D}}}\left(V_{\mathrm{G}}^{\prime}-V\right) \cdot \mathrm{d} V
$$

où $C_{\text {ox }}$ est la capacité d'oxyde par unité de surface et où $\mu_{\text {acc }}$ représente la mobilité des porteurs de la couche accumulée. La tension effective appliquée sur la grille est notée $V_{\mathrm{G}}^{\prime}$ et se définit :

$$
V_{\mathbf{G}}^{\prime}=V_{\mathrm{G}}-V_{\mathrm{FB}} \text {. }
$$

On déduit l'expression de la conductance de drain lorsque la tension drain-source tend vers zéro :

$$
\left.\frac{\partial I_{\mathrm{D}}}{\partial V_{\mathrm{D}}}\right|_{V_{\mathrm{D}} \rightarrow 0}=\frac{Z}{L_{\mathrm{eff}}} \mu_{\mathrm{acc}} \cdot C_{\mathrm{ox}} \cdot V_{\mathrm{G}}^{\prime}
$$

puis celle de la mobilité :

$$
\mu_{\mathrm{acc}}=\frac{\left.\frac{\partial I_{\mathrm{D}}}{\partial V_{\mathrm{D}}}\right|_{V_{\mathrm{D} \rightarrow 0}}}{C_{\mathrm{ox}} \cdot V_{\mathrm{G}}^{\prime}} \frac{L_{\mathrm{eff}}}{Z}
$$

qui permet de déterminer la variation de la mobilité dans la couche accumulée en fonction de la tension effective appliquée sur la grille.

A. 2 VARIATION EXPÉRIMENTALE DE LA MOBILITÉ $\mu_{\text {acc }}$ EN FONCTION DE LA TENSION DE GRILLE. - Sur les

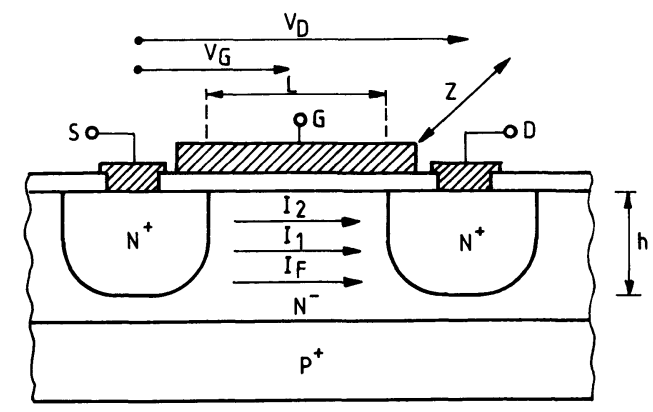

Fig. A.1. - Structure de test utilisée pour déterminer la mobilité des électrons dans la couche accumulée.

[Cross-section of an accumulation-mode $N$-channel MOS transistor.]

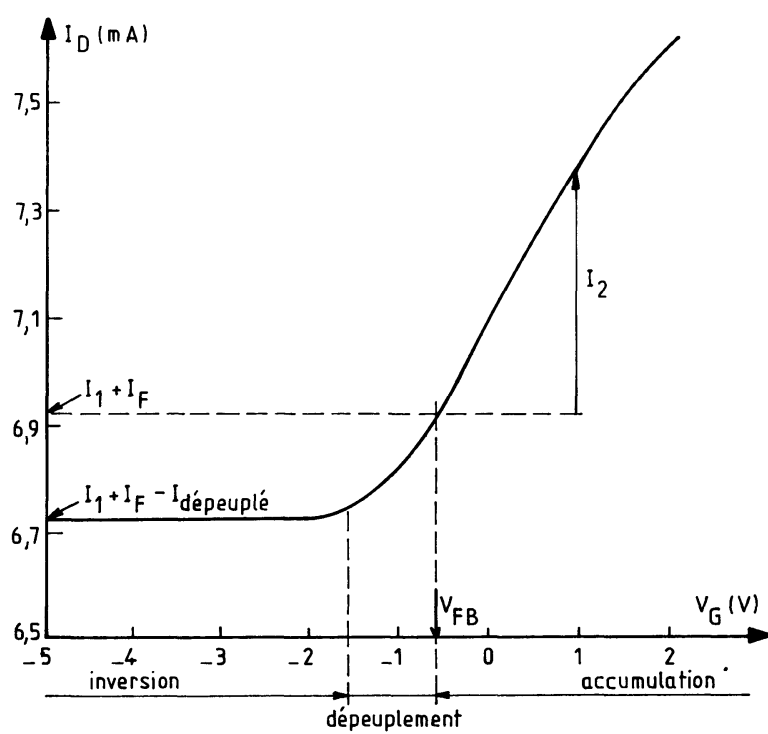

Fig. A.2. - Caractéristique $I_{\mathrm{D}}\left(V_{\mathrm{G}}\right)$ : évolution du courant suivant les trois modes successifs : inversion, dépeuplement, accumulation

$$
V_{\mathrm{D}}=100 \mathrm{mV} \quad L_{\text {eff }}=11 \mu \mathrm{m} \quad x_{0}=1000 \AA
$$

[Experimental transfer characteristic of an accumulationmode MOS transistor acting in the three successive modes : inversion, depletion, accumulation.]

caractéristiques expérimentales, on détermine tout d'abord la valeur de $V_{\text {FB }}$ - au niveau du changement de pente - la valeur de $I_{D}$ correspondante est considérée comme l'origine de la caractéristique $I_{2}\left(V_{G}^{\prime}\right)$, cette valeur vaut :

$$
I_{\mathrm{D}}\left(V_{\mathrm{G}}=V_{\mathrm{FB}}\right)=I_{1}+I_{\mathrm{F}}
$$

La caractéristique $I_{2}\left(V_{\mathrm{G}}^{\prime}\right)$ - courant drain dû à la couche accumulée en fonction de la tension de grille effective - est ensuite déterminée. Elle permet de calculer l'expression $\left.\frac{\partial I_{\mathrm{D}}}{\partial V_{\mathrm{D}}}\right|_{V_{\mathrm{D}} \rightarrow 0}$ pour différentes va- 
leurs de $V_{G}^{\prime}$, puis les variations de $\mu_{\text {acc }}$ en fonction de $V_{\mathrm{G}}^{\prime}$. Un exemple obtenu est représenté sur la courbe 5 .

\section{Annexe 2-Considérations sur le rapport périmètre sur surface.}

Dans le but d'optimiser les structures, à savoir minimiser la chute de tension à l'état passant, des études récentes $[15,16]$ ont précisé l'importance de la forme géométrique des cellules élémentaires, constituant la diffusion $P$. Il a été montré que le rapport périmètre sur surface $Z / S$ intervenant dans les différentes expressions de la conductance par unité de surface est essentiellement fonction de la densité d'intégration atteinte par le processus technologique, de la forme géométrique des cellules élémentaires et de la distance minimale $l$ à respecter entre ces cellules.

D'un point de vue théorique, il a été montré [1] que la quantité $Z / S$ atteint une valeur maximale égale à $l^{-1}$. Cette valeur est obtenue quand $r=l$ dans le cas du carré, de l'hexagone et des bandes parallèles, et pour $r=l \sqrt{3}$ dans le cas du triangle. Par suite, les quatre géométries fournissent théoriquement le même optimum pour le rapport périmètre sur surface. Des considérations plus précises, liées aux règles de dessin, montrent cependant que, en pratique, l'optimum théorique ne peut pas être atteint et que le maximum du rapport $Z / S$ se situe à $7 \%$ (triangles), $20 \%$ (hexagones), $25 \%$ (carrés), $50 \%$ (bandes parallèles) audessous de la limite théorique $l^{-1}$. Toutefois, l'avantage d'une des géométries, vis-à-vis d'une autre, est loin d'être évident sur ce seul critère. Ainsi, le triangle est affecté par des effets de " pointe ", il est par ailleurs relativement difficile à réaliser. Pour notre part, nous nous cantonnerons par la suite au concept d'optimum théorique et considérerons, artificiellement certes, que le rapport périmètre sur surface atteint la limite $l^{-1}$.

\section{Bibliographie}

[1] Rossel, P., Tranduc, H., Gamboa, M., Phan Pham, T., Limitation fondamentale dans les transistors MOS de puissance; le compromis entre la résistance à l'état passant $R_{\mathrm{ON}}$ et la tension de claquage $V_{\mathrm{DBR}}$. Revue Phys. Appl. 16 (1981) 509-515.

[2] Rossel, P., Propriétés statiques et dynamiques du transistor à effet de champ à grille isolée. Thèse de Doctorat d'Etat, No 524. Université Paul Sabatier, Toulouse, (1973).

[3] Fu, K. Y., Mobility degradation due to the gate field in the inversion layer of MOSFET'S, IEEE Electron Device Letters EDL 3 (1982) 292-293.

[4] SUN, S. C., Physics and technology of power MOSFETs, Technical Report No IDEZ 696.1, February, (1982), Stanford Electronics Laboratories.

[5] Hu, C., A parametric study of power MOSFETs, Conf. record : Power Electronics Specialists Conference, San Diego (1979).

[6] Yoshida, I., OKabe, T., Iijima, T., Ohtaka, S., NagaTA, M., A 1600 V Power MOSFET, with 20 ns Switching Speed, Proc. of the IEDM, Washington, (1983), 92-94.

[7] Ueda, D., Takagi, H., Kano, G., A New vertical double diffused MOSFET. The Self aligned Terraced Gate MOSFET, IEEE Transactions On Electron Devices ED 31 (1984) 416-420.

[8] De Moraes, B., Contribucao de optimisacao de estructura de transistors MOS de estructura nao coplanar, Ph. D. Thesis, University of Campinas, Sao Paulo, Brazil, (1982).

[9] Durand, E., Electrostatique, Tome 2, Les distributions (Masson et Cie, 1966), Chap. 4, pp. 234-267.
[10] Rossel, P., Tranduc, H., Sanchez, J. L. et BellaOUAR, A., Détermination expérimentale des paramètres des transistors MOS, Revue Phys. Appl. 18 (1983) 487-493.

[11] Sanchez, J. L., Gharbi, M., Tranduc, H. and RosSEL, P., Quasisaturation Effect in High - Voltage VDMOS Transistors IEEE Proceedings 132 (1985) 42-46.

[12] Russel, J. P., Goodman, A. M., Goodman, L. A., NeILson, J., M., The Comfet. A new high conductance MOS Gated device, IEEE Electron Devices Letters EDL 4 (1983) 63-65.

[13] Baliga, B. J., Adler, M. S., Gray, P. V., Love, R. P., The Insulated Gate Rectifier (IGR) : A new power device, switching Proc. of the IEDM 82 (1982) 264-267.

[14] Huang, J. S. T. and TAYlor, G. W., Modeling of an ion implanted Silicon gate depletion mode IGFET, IEEE Transaction on Electron Devices ED-22 (1975) 995-1001.

[15] Hower, P. L. and Geisler, M. J., Comparison of Various Source-Gate Geometries for Power MOSFETs, IEEE Transactions on Electron Devices ED-28 (1981) 1098-1101.

[16] BoArd, K., Byrne, J. and Towers, M., The Optimization of ON Resistance In Vertical DMOS Power Devices with Linear and Hexagonal Surfaces Geometries, IEEE Transactions on Electron Devices ED-31 (1984) 75-80. 